\title{
Oral Features of Systemic Sclerosis: A case-control study
}

Runnig title: Oral Features of Systemic Sclerosis

Keywords: Systemic Sclerosis, Periodontitis, Gingivitis.

Guilherme Soares Gomes da Silva, ${ }^{1}$ Marina Lins Maymone de Melo, ${ }^{1}$ Jair Carneiro Leão, ${ }^{1}$ Alessandra Tavares Carvalho ${ }^{1}$, Stephen Porter ${ }^{2}$, Angela Luzia Branco Pinto Duarte, ${ }^{3}$ Andrea Tavares Dantas, ${ }^{3}$ Luiz Alcino Monteiro Gueiros ${ }^{1 \#}$

1- Oral Medicine Unit, Departamento de Clínica e Odontologia Preventiva, Universidade Federal de Pernambuco. Recife, Brazil.

2 - UCL Eastman Dental Institute. London, UK.

3 - Rheumatology Unit, Hospital das Cínicas - Universidade Federal de Pernambuco. Recife, Brazil.

\section{\#Corresponding Author:}

Prof. Dr. Luiz Alcino Gueiros

Oral Medicine Unit. Departamento de Clínica e Odontologia Preventiva - UFPE.

Av. Prof. Moraes Rego, 1235 - CDU

Recife - PE, CEP 50670-901

+55 $812126-8816$

Email: luiz.mgueiros@ufpe.br

Conflicts of interest: none to declare

Date of submission: 01.24.2019 


\begin{abstract}
Objective: The aim of this study was to evaluate the orofacial parameters of systemic sclerosis (SSc) and its related systemic features. Subjects and Methods: A descriptive case-control study was performed from November/2015 to October/2016. Ninety-three individuals were included and divided into SSc Group $(n=50)$ and healthy controls $(C, n=43)$. Results: SSc individuals were mostly women $(43 / 50,86 \%)$, with a mean age of 46 years $( \pm 11.6$ years). Telangiectasia $(42 / 50,84 \%)$ and reduced mouth opening $(35 / 50,70 \%)$ were the most frequent orofacial findings. The periodontitis frequency was much higher in SSc individuals than in healthy controls $(90.7 \%$ x 48,83\%; p<0.001). In addition, SSc individuals presented a distinctive pattern of periodontitis, with low probing pocket depth $(2 \pm 0.65 \mathrm{~mm} \times 2 \pm 0,24 ; \mathrm{p}$ $<0.001)$, higher gingival recession $(4 \pm 2,13 \times 0,14 \pm 0,22 ; \mathrm{p}<0.001)$, higher periodontal attachment loss $(6 \pm 1,34 \mathrm{~mm} \times 2 \pm 0,43, \mathrm{p}<0.001)$ and lower gingival bleeding index values $(7.05 \pm 7.25$ x 21,57 $\pm 15,66 ; \mathrm{p}<0.001)$. Conclusions: Orofacial manifestations were common in SSc and included a unique pattern of periodontal manifestation, characterized by lower gingival bleeding index, higher periodontal attachment loss and low probing depth.
\end{abstract}

Key words: Systemic sclerosis; periodontal disease; orofacial features. 


\section{INTRODUCTION}

Systemic sclerosis (SSc) is an autoimmune multisystemic rheumatic disease of unknown etiology, characterized by three pathophysiological processes: obstructive vasculopathy of the small vessels, immunological deregulation followed by fibroblast inflammation and dysfunction, leading to an increased extracellular matrix deposition (Baron, 2015). Inflammatory, fibrotic, and atrophic changes characteristically lead to thickening of the skin, a key finding of the disease. In addition, fibrosis of internal organs, particularly the lungs, gastrointestinal tract, heart, and kidneys, promote important functional limitations and significant morbidity-mortality (Vonk et al, 2009).

Oral involvement of SSc is associated with varying degrees of functional disability. A wide range of orofacial manifestations are observed and include rigidity and atrophy of facial skin, mask-like facial appearance, telangiectasia, loss of elasticity of the oral mucosa, hardening of the tongue and soft palate, varying degrees of xerostomia, periodontitis, that ultimately lead to limitations in chewing, speaking and swallowing (Wood \& Lee, 1988; Crincoli, Fatone et al., 2016). Progressive fibrosis of the facial muscles, lips, and cheeks, as well as the furrowing of the perioral grooves can lead to reduced mouth opening (Yuen et al., 2012). Periodontal tissues are also frequently altered in SSc, presenting with attachment loss, scarce bleeding on probing and widening of periodontal space (Jagadish et al., 2012). Besides, SSc-related oral limitations and hand deformities may preclude individual and professional oral hygiene measures (Pischon et al. 2016). The salivary glands are also involved due to fibrotic alterations, which may cause severe hyposalivation and considerable damage to the oral functions (Yenisey, Külünk et al. 2005). Also, about $1 / 3$ of the SSc patients develop with associated Sjögren's syndrome $(\mathrm{SSj})$ and may present autoantibodies related to both diseases (Kobak, Oksel et al. 2013).

Nevertheless, data about the head and neck involvement of SSc are still scarce and have not been adequately categorized according to disease type. Moreover, some patterns of orofacial involvement have not been clearly described, leading to a limited understanding of the clinical significance of orofacial alterations in SSc. In this context, the aim of this study was to evaluate the orofacial parameters, the severity of periodontal disease, and hyposalivation of individuals with SSc, comparing them among the limited and diffuse cutaneous clinical forms.

\section{SUBJECTS AND METHODS}




\section{Ethical aspects}

The Research Ethics Committee of the Universidade Federal de Pernambuco approved this study under protocol $\mathrm{N}^{\circ}$ (49049415.1.0000.5208). The term of free and informed consent was signed before inclusion in the study. All the individuals were duly informed about the nature of the study, its risks and benefits.

\section{Study Design, Population Studied}

A descriptive case-control study was conducted with individuals diagnosed with SSc evaluated at the Rheumatology Unit/ Systemic Sclerosis Outpatient Clinic of the Hospital das Clinicas - UPFE from November 2015 to October 2016. Individuals of both sexes, older than 18 years and diagnosed with SSc were eligible for the study. Individuals were evaluated by a rheumatologist and classified according to the ACR/EULAR classification criteria (Van Den Hoogen, Khanna et al. 2013). Patients presenting with pregnancy, localized forms of SSc, and other rheumatologic autoimmune diseases were excluded from the study. In order to evaluate the periodontal profile, a control group was composed of 43 healthy dentate individuals, matched by sex and age, who reported no autoimmune disease, history of smoking, use of antibiotics during the last six months prior the examination, pregnancy or diabetes. Control group was composed of individuals accompanying the SSc patients to the rheumatology unit, sharing a similar social and educational background.

Cutaneous involvement was evaluated on inclusion in the study by means of the modified Rodnan Skin Score. This clinical evaluation standardized for SSc patients and uses a subjective system that evaluates 17 spots of the skin, including fingers, hands, forearms, arms, feet, legs, thighs, face, chest and abdomen, and assigning each spot with a score ranging from 0 (no sclerosis), 1 (mild sclerosis), 2 (moderate sclerosis where the skin cannot be pinched) and 3 (severe sclerosis - skin without mobility). The Rodnan score was calculated by summing the values, ranging from zero to 51 (Clements, Lachenbruch et al. 1995). All patients were evaluated by the same examiner, a rheumatologist experienced in the management of SSc. The patients were classified as having limited cutaneous or diffuse cutaneous clinical forms according to the criteria of LeRoy and Medsger (2001).

For the evaluation of associated $\mathrm{SSj}$, patients were classified according to the AmericanEuropean Consensus Group (Vitali, Bombardieri et al., 2002). For this purpose, complaints of xerophthalmia and xerostomia were evaluated, in addition to the resting salivary flow, Schirmer test, and biopsy of the minor salivary glands. 


\section{Clinical Oral Evaluation}

The patients answered a structured questionnaire to obtain clinical data, including the sociodemographic, medical, present history of the disease, and orofacial conditions. The medical data were complemented by evaluating the clinical record charts. After this, the patients underwent a thorough oral evaluation, including periodontal assessment and determination of the DMFT index (World Health 2013). Dental erosion was also evaluated. For this study, dental erosion was is considered as the loss of enamel characterization, generating an appearance of extreme smoothness and gloss (Ganss \& Lussi, 2006; Lussi \& Carvalho, 2014).

The interincisal distance was evaluated and recorded through a digital pachymeter, based on the distance of the edges of the maxillary and mandibular central incisors after the maximum opening of the oral cavity. Edentulous patients who did not wear prosthesis were not evaluated. (Silvestre-Rangil, Martinez-Herrera et al., 2015).

The resting sialometry test and Schirmer test were performed during the morning, as previously described (Oliveira, de Souza et al., 2015). Minor salivary gland biopsy was performed in patients with suspected $\mathrm{SSj}$; microscopic evaluation was positive when the focus score was greater than 1 . The focus score was determined by counting the number of foci (50 or more lymphocytes $/ 4 \mathrm{~mm}^{2}$ ) in relation to the total glandular area. (Daniels, Cox et al., 2011). Crenation was determined with the persistent presence of marks left by the outline of the teeth on the jugal mucosa (Ghom and Ghom 2014).

The same periodontist performed periodontal examinations. A manual periodontal probe (PCP 11; Hu-Friedy®) was used, and the readings were recorded to the nearest one millimeter $(\mathrm{mm})$. All periodontal measurements were assessed at four sites of each tooth (mesiobuccal, distobuccal, mesiolingual, and distolingual), as described before (Pischon, Pischon et al., 2008; Kaur, White et al., 2013). The gingival bleeding index was evaluated, according to Carter and Barnes (1974).

Pocket probing depth was defined as the distance from the free gingival margin to the bottom of the sulcus or periodontal pocket; The periodontal attachment loss (PAL) was defined as the distance from the cement-enamel junction to the bottom of the sulcus or periodontal pocket and was calculated as the sum of probing depth and gingival recession measurements; And the Gingival recession was defined as the distance from the cemento-enamel junction to the free gingival margin (Ainamo \& Bay 1975). The data described were noted on a specific periogram for tabulation and statistical analysis. For the severity and extent of periodontal 
disease, the American Academy of Periodontology 2015 was used. According to the severity of the periodontal disease, this was classified as: $\mathrm{PD}=4 \mathrm{~mm}$ (light); $\geq 5$ and $<7 \mathrm{~mm}$ (moderate); $\geq 7 \mathrm{~mm}$ (Severe), with clinical attachment loss, and bleeding on probing also being taken into consideration. The extent of periodontal disease was classified as localized and generalized; being characterized as localized when $\leq 30 \%$ of the sites were involved, and generalized when $>30 \%$ of the sites were involved (Geurs N 2015).

\section{$\underline{\text { Statistical Analysis }}$}

Descriptive statistics were used to sum up the initial characteristics of the sample. The samples were tested for normality by the Shapiro-Wilk test, and data were shown to be nonparametric. Categorical data were evaluated by the Exact Fisher and Chi-square tests. The odds ratio and confidence interval at $95 \%$ were determined for the statistically significant results. Numerical variables were evaluated by the Mann-Whitney test. To evaluate correction between the data were the Spearman correlation test. The level of significance considered was 5\%. Analyses were performed using Prism GraphPad 6.0.

\section{RESULTS}

\section{Study subjects}

The study included 50 individuals with SSc; $43 / 50$ women $(86 \%$, female to male ratio $=6.14: 1)$ with a mean age of 46 years $( \pm 11.6$ years). Control group was composed of 43 individuals, $36 / 43$ women $(84 \%$, female to male ratio $=5.14: 1)$, with a mean age of 44 years $( \pm$ 8 years). The diffuse cutaneous form was slightly more frequent $(26 / 50,52 \%)$ and $36 / 50(72 \%)$ individuals were diagnosed for more than five years. Most of the patients presented with esophageal and pulmonary involvement, as well as digital ulcers and Raynaud's phenomenon. The clinical features of the patients are described in (Table 1).

\section{Periodontal disease}

Periodontitis was more frequent in SSc $(C, n=21 ; \operatorname{SSc}, n=39 ; \mathrm{p}<0.001)$. Also, generalized periodontitis was more common in SSc individuals (19/39, 44.2\%, p=0.13). Severe periodontitis was the most frequent presentation in SSc patients (17/43, 39.5\%). Probing depth and gingival bleeding index values were significantly reduced $(\mathrm{p}<0.001)$ and clinical attachment loss was significantly elevated in SSc $(p<0.001)$ (Tables 2$)$. It could be observed 
some thick fibrotic deposition on the gingiva and alveolar mucosa (Fig.1).

\section{Orofacial Features}

Telangiectasia $(42 / 50,84 \%)$, reduced mouth opening $(36 / 50,72 \%)$ and buccal mucosa crenation $(31 / 50,62 \%)$ were the most frequent orofacial findings. It could also be noted a high frequency of tongue depapillation $(18 / 50,36 \%)$. Likewise, severe periodontitis presented a higher frequency in dcSSc $(11 / 24,44.83 \%)$ than in $\operatorname{lcSSc}(06 / 26,23.07 \%)$ ( $\mathrm{p}=0.078)$. No differences were observed in salivary flow, interincisal distance, number of missing teeth, and filled teeth in relation to clinical forms of SSc $(\mathrm{p}>0.05)$ (Table 3$)$.

These oral features were not associated with the systemic manifestations of SSc described in table 1 (data not shown). There was no association between the use of immunosuppressant agents and the clinical form of SSc $(\mathrm{p}=0.423)$. Limitation of dental brushing was a common complaint (52\%), but assistance with brushing was not commonly reported (14\%) (Table 3).

\section{Sjögren's syndrome}

The diagnosis of SSj was demonstrated in Table 4. Clinical evaluation of SSj, Twentyfour patients presented clinical suspicion of $\mathrm{SSj}$ based on reduced results of sialometry/Schirmer tests; $16 / 50$ patients $(32 \%)$ were diagnosed with SSj. Salivary gland biopsy was performed in 10 patients, of whom 04 presented a focus score higher than one. All SSj-related clinical features were different between SSc and SSc/SSj individuals (Table 4). The SSc/SSj patients had higher Rodnan scores $(24.31 \pm 16)$ than SSc individuals without SSj (15.8 $\pm 13)(\mathrm{p}=0.03)$. No correlation was found between the Rodnan score and stimulated salivary flow $(\mathrm{SSF})(\mathrm{p}=0,343)$, resting Salivary flow $(\mathrm{RSF})(\mathrm{p}=0.231)$, mouth opening $(\mathrm{p}=0.210)$, reduced mouth opening $(\mathrm{p}=0.359)$, crenation of the oral mucosa $(\mathrm{p}=0.359)$, periodontitis $(\mathrm{p}$ $=0.226)$, difficulty of brushing the teeth $(\mathrm{p}=0.166)$ and need of help to brush the teeth $(\mathrm{p}=$ 0.307) (Table 4). 


\section{DISCUSSION}

This study described the clinical orofacial characteristics of a group of 50 patients with systemic sclerosis; these findings were described according to the clinical form of the disease. Periodontal disease in the patients with SSc presented a distinct clinical profile, characterized by high clinical attachment loss and low bleeding on probing values. On the other hand, periodontal pocket depth, bleeding on probing, and gingival bleeding index values were diminished in SSc patients. In addition, reduced mouth opening, telangiectasia, buccal mucosa crenation, as well as Sjögren's syndrome, were commonly observed among SSc individuals.

Studies have pointed out a close relationship between periodontal disease and SSc (Jagadish et al., 2012; Baron et al., 2015; Crincoli et al., 2016; Pischon et al., 2016). The present study observed periodontitis in more than $90 \%$ of SSc individuals, associated with a low gingival bleeding index. It could also be observed a significantly higher periodontal attachment loss, periodontal pocket depth, and gingival recession in the SSc subjects. Similar findings regarding periodontal attachment loss and periodontal pocket depth were observed by Pischon, Hoedke et al (2016), reinforcing the idea that these periodontal aspects characterize the oral involvement of SSc, together with low bleeding on probing. Nevertheless, Leung et al (2011) and Isola et al (2017) observed a higher incidence of bleeding on probing in SSc patients, suggesting a possible interference of other factors in periodontal disease presentation.

With the purpose of explaining the predisposition to the periodontal disease of SSc individuals, Baron et al (2015) evaluated the relationship between periodontal status and hyposalivation, interincisal distance, sclerodactyly, and the Health Assessment Questionnaire (HAQ), but no association was observed. Similarly, the present study also failed to observe the influence of orofacial manifestations and functional limitations in the frequency of periodontal disease. Nevertheless, gingival inflammation in individuals with SSc was associated with manual dexterity for performing oral hygiene and the use of dental floss at night (Yuen et al., 2014). Moreover, gingivitis was reported to be more frequent among patients with the diffuse cutaneous disease, and its frequency can be influenced by reduced mouth opening, higher Rodnan scores and limited manual dexterity (Poole et al., 2005). In this context, these aspects can be considered risk factors for worst oral outcomes in SSc individuals. Interestingly, we found that difficulty with oral hygiene was a common complaint in SSc patients, but it was not associated with periodontal status.

Among the oral hallmarks of SSc, reduced mouth opening is possibly the most common and well known (Pizzo et al., 2003; Wada \& Ram, 2013; Crincoli et al., 2016; Rannou et al., 2016; Jung et al., 2017). Baldrighi et al (2016) observed that diminished mouth opening of SSc 
patients was associated with limited elasticity of the oral mucosa, skin atrophy, collagen deposition on the peri-oral tissues as well as the pathological resorption of the condylar processes. Due to the diminished interincisal distance and increased rigidity of the buccal mucosa, oral hygiene routine is often impaired, as well as oral examination and dental treatment. So this aspect should be taken into consideration to form the clinical oral scenario and periodontal risk pattern of SSc.

Another very common oral features of SSc included telangiectasia and crenation of the buccal mucosa, and the present study showed a similar distribution of these aspects between the two clinical forms of SSc. Telangiectasia was also reported to affect more than $80 \% \mathrm{SSc}$ individuals from a Chinese study (Leung et al., 2011), similar to the present findings. Additionally, Eversole et al (1984) was the first to describe crenation of the buccal mucosa as a clinical pattern of SSc, reported in of $26 \%$ of his SSc sample. Mucosal fibrosis, together with hyposalivation may act to increase the friction between the teeth and buccal mucosa, favoring the establishment of crenation.

The presence of SSj was demonstrated in $32 \%$ of the present patients, similarly to other studies (Kobak et al., 2013). We demonstrated that patients with SSc/SSj presented lower stimulated and unstimulated salivary flow rates, as well as reduced Schirmer test when compared to patients with SSc and no SSj. In addition, salivary gland biopsy was positive in a fraction of the patients with $\mathrm{SSc} / \mathrm{SSj}$ (40\%), which is similar to the context of primary $\mathrm{SSj}$ (38.78\% of the patients with suspected primary SSj) (Daniels, Cox et al., 2011). We could observe some areas of fibrosis in the salivary gland parenchyma, usually with scarce inflammatory infiltrate, although Kucuk et al. (2018) reported that patients with both SSc and SSj present less plasma cell infiltration, and a similar amount of fibrosis than individuals with primary SS. It should be further evaluated whether some patients with SSc that do not fulfill SSj criteria actually develop hyposalivation secondary to glandular fibrosis. Moreover, we observed that Rodnan score was elevated among SSc/SSj (SSc) patients ( $p=0.03$ ), favoring the rationale that a more extensive cutaneous involvement can be associated not only with a more significant systemic condition but also with reduced salivary gland function.

To the best of our knowledge, this is the first study to report a high prevalence of dental erosion among SSc individuals, observed in nearly one-third of the included patients. There are some pieces of evidence supporting the association between gastroesophageal reflux, hyposalivation and dental erosion in children, thus reinforcing this association (Linnett et al., 2002; Yoshikawa et al., 2012). Baron et al. (2015) observed that SSc individuals with gastroesophageal reflux present higher dental loss, but dental erosion was not evaluated. 
Interestingly, we did not find any association between esophageal involvement and dental erosion and dietary habits should be considered as a possible cause of dental erosion among SSc patients.

Certain limitations of this study are understood, among them the limited number of patients $(n=50)$. Nevertheless, the rarity of the disease and the specificity of the clinical parameters observed should be taken into consideration, making it more relevant to compare them among the clinical forms of the disease. Given this, we were unable to generalize the data here presented due to the low epidemiological value. This research was conducted in a singlecenter, which limited access to a more significant number of patients. Moreover, a case-control design with descriptive purposes was used, which did not make it possible to evaluate cause and effect, and limited a better understanding of the pathogenesis of orofacial changes in SSc.

According to the results of this study, patients with systemic sclerosis presented a high prevalence of the periodontal disease. The presence of telangiectasia, trismus, and buccal mucosa indentation were common findings in SSc, irrespective of the clinical type. The diagnosis of $\mathrm{SSj}$ is associated with greater cutaneous involvement in SSc. A higher frequency of periodontitis was found in the diffuse cutaneous form. Low bleeding on probing, normal probing depth associated with a high prevalence of gingival recessions, characterized the periodontitis in SSc, suggesting that gingival fibrosis must be considered in the diagnosis of periodontal disease in this group of individuals. Based on these findings, oral involvement is common in SSc and periodontists, and oral medicine practitioners should be aware that bleeding is not a hallmark of periodontal involvement, so clinical and personal oral care should be adapted to each patient needs. 


\section{REFERENCES}

Ainamo, J., \& Bay, I. (1975). Problems and proposals for recording gingivitis and plaque. International dental journal, 25 (4), 229-235.

Baldrighi, S. E. Z. D. M., Almeida, L. F., Alves, C. S., Silva, A. K. A. D., Barros Filho, L., Macieira, J. C., \& César, C. P. H. A. R. (2016). Amplitude máxima da abertura de boca na esclerose sistêmica. Distúrbios da Comunicação, 28(1).

Baron, M., Hudson, M., Tatibouet, S., Steele, R., Lo, E., Gravel, S., ... Masetto, A. (2015). Relationship between disease characteristics and orofacial manifestations in systemic sclerosis: Canadian Systemic Sclerosis Oral Health Study III. Arthritis care \& research, 67(5), 681-690. doi: 10.1002/acr.22490.

Carter, H. G., \& Barnes, G. P. (1974). The gingival bleeding index. Journal of Periodontology, 45(11), 801-805. doi.org/10.1902/jop.1974.45.11.801

Clements, P. H., Lachenbruch, P., Siebold, J., White, B., Weiner, S., Martin, R., ... Collier, D. (1995). Inter and intraobserver variability of total skin thickness score (modified Rodnan TSS) in systemic sclerosis. The Journal of Rheumatology, 22(7), 1281-1285.

Crincoli, V., Fatone, L., Fanelli, M., Rotolo, R. P., Chialà, A., Favia, G., \& Lapadula, G. (2016). Orofacial manifestations and temporomandibular disorders of systemic scleroderma: an observational study. International Journal of Molecular Sciences, 17(7), 1189. doi.org/10.3390/ijms17071189

Daniels, T. E., Cox, D., Shiboski, C. H., Schiødt, M., Wu, A., Lanfranchi, H., ... De Souza, Y. (2011). Associations between salivary gland histopathologic diagnoses and phenotypic features of Sjögren's syndrome among 1,726 registry participants. Arthritis \& Rheumatism, 63(7), 20212030. doi.org/10.1002/art.30381

Eversole, L. R., Jacobsen, P. L., \& Stone, C. E. (1984). Oral and gingival changes in systemic sclerosis (scleroderma). Journal of periodontology, 55(3), 175-178.

Ganss, C. \& Lussi A. (2006). Diagnosis of erosive tooth wear. Dental Erosion, 20, 32-43. Karger Publishers. doi.org/10.1159/000093349

Geurs, N., Iacono, V., Krayer, J., Mealey, B. L., Paquette, D., Pearson, B., ... Schweinebraten, M. (2015). American Academy of Periodontology Task Force report on the update to the 1999 classification of periodontal diseases and conditions. Journal of Periodontology, 86(7), 835838. doi.org/10.1902/jop.2015.157001

Ghom, A. G., \& Ghom, S. A. L. (Eds.). (2014). Textbook of oral medicine. JP Medical Ltd.

Jagadish, R., Mehta, D. S., \& Jagadish, P. (2012). Oral and periodontal manifestations associated with systemic sclerosis: A case series and review. Journal of Indian Society of Periodontology, 16(2), 271. doi: 10.4103/0972-124X.99275

Jung, S., Martin, T., Schmittbuhl, M., \& Huck, O. (2017). The spectrum of orofacial manifestations in systemic sclerosis: a challenging management. Oral diseases, 23(4), 424-439. doi.org/10.1111/odi.12507 
Kaur, S., White, S., \& Bartold, P. M. (2013). Periodontal disease and rheumatoid arthritis: a systematic review. Journal of Dental Research, 92(5), 399-408. doi.org/10.1177/0022034513483142

Kobak, S., Oksel, F., Aksu, K., \& Kabasakal, Y. (2013). The frequency of sicca symptoms and $\mathrm{S}$ jögren's syndrome in patients with systemic sclerosis. International journal of rheumatic diseases, 16(1), 88-92. doi.org/10.1111/j.1756-185X.2012.01810.x

Kucuk, U., Sarioglu, S., Cetin, P., Sari, I., \& Birlik, M. (2018). Histopathological differences between primary Sjögren's syndrome and Sjögren's syndrome accompanied by scleroderma. Indian Journal of Pathology and Microbiology, 61(3), 319. doi: 10.4103/IJPM.IJPM_416_17

LeROY, E. C., \& Medsger Jr, T. A. (2001). Criteria for the classification of early systemic sclerosis. Journal of Rheumatology, 28(7), 1573-1576.

Leung, W. K., Chu, C. H., Mok, M. Y., Yeung, K. S., \& Ng, S. K. (2011). Periodontal status of adults with systemic sclerosis: case - control study. Journal of periodontology, 82(8), 11401145. doi.org/10.1902/jop.2010.100593

Lussi, A., \& Carvalho, T. S. (2014). Erosive tooth wear: a multifactorial condition of growing concern and increasing knowledge. Erosive Tooth Wear, 25,1-15. Karger Publishers. doi.org/10.1159/000360380

Linnett, V., Seow W.K., Connor, F., Shepherd, R. (2002). Oral health ofchildren with gastroesophageal reflux disease: a controlled study. Aust Dent J, 47(2),156-162. doi.org/10.1111/j.1834-7819.2002.tb00321.x

Oliveira, H. F., de Souza, T. R., Carvalho, C. N., Duarte, A., Carvalho, A. T., Leão, J. C., \& Gueiros, L. A. (2015). Serologic profile and clinical markers of Sjögren syndrome in patients with rheumatoid arthritis. Oral surgery, oral medicine, oral pathology and oral radiology, 119(6), 628-635. doi.org/10.1016/j.oooo.2015.02.479

Pischon, N., Hoedke, D., Kurth, S., Lee, P., Dommisch, H., Steinbrecher, A., Pischon, T., ... Detert, J. (2016). Increased periodontal attachment loss in systemic sclerosis patients. J. Periodontol, 28, 1-12. doi: 10.1902/jop.2016.150475.

Pischon, N., Pischon, T., Kröger, J., Gülmez, E., Kleber, B. M., Bernimoulin, J. P., ... Detert, J. (2008). Association among rheumatoid arthritis, oral hygiene, and periodontitis. Journal of periodontology, 79(6), 979-986. doi.org/10.1902/jop.2008.070501

Poole, J. L., Brewer, C., Rossie, K., Good, C. C., Conte, C., \& Steen, V. (2005). Factors related to oral hygiene in persons with scleroderma. International journal of dental hygiene, 3(1), 1317. doi.org/10.1111/j.1601-5037.2004.00108.x

Rannou, F., Boutron, I., Mouthon, L., Sanchez, K., Tiffreau, V., Hachulla, E., \& Poiraudeau, S. (2016). A personalized physical therapy program or usual care for patients with systemic sclerosis: A randomized controlled trial. Arthritis Care Res. (Hoboken) 
Silvestre-Rangil, J., Martinez-Herrera, M., \& Silvestre, F. J. (2015). Dental management of patients with microstomia. A review of the literature and update. Journal of Oral Research, 4(5), 340-350. doi: 10.17126/JORALRES.2015.065

Vonk, M. C., Broers, B., Heijdra, Y. F., Ton, E., Snijder, R., van Dijk, A. P. J., ... van Daele, P. L. A. (2009). Systemic sclerosis and its pulmonary complications in The Netherlands: an epidemiological study. Annals of the rheumatic diseases, 68(6), 961-965. doi: 10.1136/ard.2008.091710

Van Den Hoogen, F., Khanna, D., Fransen, J., Johnson, S. R., Baron, M., Tyndall, A., ... Riemekasten, G. (2013). 2013 classification criteria for systemic sclerosis: an American College of Rheumatology/European League against Rheumatism collaborative initiative. Arthritis \& Rheumatism, 65(11), 2737-2747. doi.org/10.1002/art.38098

Vitali, C., Bombardieri, S., Jonsson, R., Moutsopoulos, H. M., Alexander, E. L., Carsons, S. E., ... Weisman, M. (2002). Classification criteria for Sjögren's syndrome: a revised version of the European criteria proposed by the American-European Consensus Group. Annals of the rheumatic diseases, 61(6), 554-558. doi: 10.1136/ard.61.6.554

Wood, R. E., \& Lee, P. (1988). Analysis of the oral manifestations of systemic sclerosis (scleroderma). Oral surgery, oral medicine, oral pathology, 65(2), 172-178. doi.org/10.1016/0030-4220(88)90161-2

World Health Organization. (2013). Oral health surveys: basic methods. World Health Organization.

Yenisey, M., Külünk, T., Kurt, Ş., \& Ural, C. (2005). A prosthodontic management alternative for scleroderma patients. Journal of oral rehabilitation, 32(9), 696-700. doi.org/10.1111/j.13652842.2005.01474.x

Yuen, H. K., Weng, Y., Reed, S. G., Summerlin, L. M., \& Silver, R. M. (2014). Factors associated with gingival inflammation among adults with systemic sclerosis. International journal of dental hygiene, 12(1), 55-61. doi.org/10.1111/idh.12024

Yoshikawa, H., Furuta, K., Ueno, M., Egawa, M., Yoshino, A., Kondo, S., ... Sekine, J. (2012). Oral symptoms including dental erosion in gastroesophageal reflux disease are associated with decreased salivary flow volume and swallowing function. Journal of gastroenterology, 47(4), 412-420. doi: 10.1007/s00535-011-0515-6. 
Table 1. Clinical form, time of diagnosis and systemic involvement of patients with systemic sclerosis $(\mathbf{n}=\mathbf{5 0})$.

\begin{tabular}{|c|c|}
\hline Characteristics & $\mathbf{N}(\%)$ \\
\hline \multicolumn{2}{|l|}{ Clinical form of SSc } \\
\hline Limited cutaneous & $24(48 \%)$ \\
\hline Early & $05(20,83 \%)$ \\
\hline Established & $19(79,16 \%)$ \\
\hline Diffuse cutaneous & $26(52 \%)$ \\
\hline Early & $00(00 \%)$ \\
\hline Established & $26(100 \%)$ \\
\hline \multicolumn{2}{|l|}{ Time of diagnosis } \\
\hline$<2$ years & $02(4 \%)$ \\
\hline $2-5$ years & $12(24 \%)$ \\
\hline$>5$ years & $36(72 \%)$ \\
\hline Cutaneous involvement & $48(96 \%)$ \\
\hline Esophageal involvement & $47(94 \%)$ \\
\hline Digital ulcers & $32(64 \%)$ \\
\hline Interstitial lung disease & $32(64 \%)$ \\
\hline Raynaud's Phenomenon & $28(56 \%)$ \\
\hline Pulmonary arterial hypertension & $13(26 \%)$ \\
\hline Myopathy & $13(26 \%)$ \\
\hline Arthritis & $04(8 \%)$ \\
\hline Renal crisis & $01(2 \%)$ \\
\hline
\end{tabular}


Table 2. Evaluation of periodontal features in SSc patients and controls.

\begin{tabular}{|c|c|c|c|}
\hline Periodontal features ${ }^{\dagger}$ & Control $\dagger$ & SSct & p-value** \\
\hline & $\mathrm{n}(\%) *$ & $\mathrm{n}(\%)^{*}$ & \\
\hline PPD (mm); Mean \pm sd & $2 \pm 0.24$ & $2 \pm 0.65$ & $\mathrm{P}<0.001$ \\
\hline GR $(\mathbf{m m}) ;$ Mean \pm sd & $0.14 \pm 0.22$ & $4 \pm 2.13$ & $\mathrm{P}<0.001$ \\
\hline PAL $(\mathbf{m m}) ;$ Mean \pm sd & $2 \pm 0.43$ & $6 \pm 1.34$ & $\mathrm{P}<0.001$ \\
\hline Severity categories of periodontitis & $21(48.83 \%)$ & $39(90.7 \%)$ & \\
\hline $\begin{array}{l}\text { no periodontitis } \\
\text { mild periodontitis }\end{array}$ & $\begin{array}{l}22(51.16 \%) \\
12(27.90 \%)\end{array}$ & $\begin{array}{l}04(9.3 \%) \\
08(18.6 \%)\end{array}$ & $\begin{array}{c}\mathrm{P}<0.001 \\
\mathrm{OR}=10.21 \\
\mathrm{IC} 95 \% \\
(3.11-33.59)^{* * *}\end{array}$ \\
\hline moderate periodontitis & $07(16.27, \%)$ & $14(32.6 \%)$ & \\
\hline severe periodontitis & $2(4.65 \%)$ & $17(39.5 \%)$ & \\
\hline $\begin{array}{c}\text { Extension categories of periodontitis } \\
\text { Generalized }\end{array}$ & $06(22.67 \%)$ & $19(44.2 \%)$ & $\mathrm{P}=0.13$ \\
\hline Localized & $15(77.33 \%)$ & $20(46.5 \%)$ & \\
\hline GBI (\%); Mean \pm sd & $21.57 \pm 15.66$ & $7.05 \pm 7.25$ & $\mathrm{P}<0.001$ \\
\hline
\end{tabular}

sd - standard deviation

† SSc $(n=43)$ and control subjects $(n=43)$.

* Data are numbers of persons (percentages) unless stated otherwise.

**Variables were compared between cases and controls using the chi-square test or Fishers exact test for categorical variables and using Wilcoxon-Mann-Whiney test for continuous variables without normal distribution.

PPD (periodontal pocket depth), GR (gingival recession), PAL (Periodontal attachment loss), GBI (gingival bleeding index).

*** No periodontitis x Mild/moderate/severe periodontitis 
Table 3. Orofacial characteristics and diagnosis of Sjögren's syndrome in an overview, and according to the clinical type of systemic sclerosis.

\begin{tabular}{|c|c|c|c|c|}
\hline Characteristics & $\begin{array}{c}\text { SSc } \\
\text { n (\%) }\end{array}$ & $\begin{array}{l}\text { dcSSc } \\
\text { n }(\%)\end{array}$ & $\begin{array}{l}\text { lcSSc } \\
\text { n (\%) }\end{array}$ & p-value \\
\hline Orofacial telangiectasia & $42(84 \%)$ & $23(89 \%)$ & $19(79 \%)$ & 0.456 \\
\hline Reduced mouth opening & $36(72 \%)$ & $20(77 \%)$ & $16(62 \%)$ & 0.284 \\
\hline Buccal mucosa crenation & $31(62 \%)$ & $16(62 \%)$ & $15(62 \%)$ & 1.0 \\
\hline Tongue Depapillation & $18(36 \%)$ & $08(31 \%)$ & $10(42 \%)$ & 0.733 \\
\hline Dental erosion $\dagger$ & $12(28 \%)$ & $06(23 \%)$ & $06(25 \%)$ & $0.744^{*}$ \\
\hline Severity categories of periodontitis $\dagger$ & & & & $0.078 *$ \\
\hline No Periodontal disease & $04(9.3 \%)$ & $00(0.0 \%)$ & $04(16.6 \%)$ & \\
\hline Mild periodontitis & $08(18.6 \%)$ & $06(23.07 \%)$ & $02(8.3 \%)$ & \\
\hline Moderate periodontitis & $14(32.6 \%)$ & $08(30.76 \%)$ & $06(25 \%)$ & \\
\hline Severe periodontitis & $17(39.5 \%)$ & $11(44.83 \%)$ & $06(23.07 \%)$ & \\
\hline Extension categories of periodontitis $†$ & & & & $0.263 *$ \\
\hline Generalized & $19(44.2 \%)$ & $11(42.30 \%)$ & $08(33.33 \%)$ & \\
\hline Localized & $20(46.5 \%)$ & $12(46.15 \%)$ & $08(33.33 \%)$ & \\
\hline Limitation to dental brushing & $26(52 \%)$ & $13(50 \%)$ & $13(54.16 \%)$ & $0.156^{* *}$ \\
\hline Assistance to dental brushing & $07(14 \%)$ & $03(11.53 \%)$ & $04(16.6 \%)$ & $0.263 * *$ \\
\hline Interincisal distance $(\mathrm{mm}) \dagger$ Mean $\pm \mathrm{sd}$ & $33.1 \pm 8.12$ & $34.74 \pm 9.1$ & $31.63 \pm 6.8$ & $0.23 * *$ \\
\hline Salivary flow; Mean \pm sd & $0.74 \pm 0.58$ & $0.73 \pm 0.57$ & $0.75 \pm 0.59$ & $0.95 * *$ \\
\hline Number of teeth lost $\uparrow$ Mean \pm sd & $10.5 \pm 8.78$ & $10.29 \pm 9.6$ & $10.69 \pm 8.1$ & $0.56 * *$ \\
\hline Number of teeth filled $†$ Mean \pm sd & $5.24 \pm 5.69$ & $4.53 \pm 5.21$ & $6 \pm 6.08$ & $0.48 * *$ \\
\hline Number of teeth decayed $\dagger$ Mean \pm sd & $1.08 \pm 1.67$ & $1.30 \pm 2.01$ & $0.83 \pm 1.14$ & $0.36 * *$ \\
\hline Sjögren's Syndrome & $16(32 \%)$ & $10(38.46 \%)$ & $6(25 \%)$ & $0.135^{* *}$ \\
\hline
\end{tabular}

\section{$\mathrm{Sd}$ - Standard deviation}

$\dagger 07$ patients total edentulous / * Fishers exact test / ** Wilcoxon-Mann-Whiney test 
Table 4. Clinical parameters evaluated for the diagnosis of Sjögren's syndrome.

\begin{tabular}{lccc}
\hline Characteristic & SSc/No SSj (n=34) & SSc/SSj (n=16) & TOTAL \\
\hline Ocular symptoms (+) & $5(14.70 \%)$ & $16(100 \%)$ & $21(42 \%)$ \\
Schirmer Test (+) & $5(14.70 \%)$ & $15(93.75 \%)$ & $20(40 \%)$ \\
Schirmer Test; Mean \pm SD & $13.82 \pm 10.84$ & $3.56 \pm 8.18$ & $10.26 \pm 10.90$ \\
Oral symptoms (+) & $5 / 34(14.70 \%)$ & $15(93.75 \%)$ & $20(40 \%)$ \\
Resting Salivary flow (+) & $5 / 34(14.70 \%)$ & $15(93.75 \%)$ & $20(40 \%)$ \\
Resting Salivary flow; Mean \pm SD & $0.94 \pm 0.59$ & $0.32 \pm 0.16$ & $0.74 \pm 0.58$ \\
Stimulated Salivary flow; Mean \pm SD & $2.41 \pm 2.25$ & $0.88 \pm 0.87$ & $1.94 \pm 1.48$ \\
Biopsy of minor salivary glands (+) & $0 / 6(0 \%)$ & $4 / 10(40 \%)$ & $4 / 16(25 \%)$ \\
\hline
\end{tabular}

$\mathrm{SSc}-$ Systemic sclerosis; SSj Sjögren's syndrome Fischer exact test. $\mathrm{P}=0.05$. 


\section{Figure legends}

Figure 1. Extensive fibrosis in the gingiva and alveolar mucosa of an individual with cutaneous diffuse systemic sclerosis. 